Review Article

\title{
Analysis of Drag Reduction Methods and Mechanisms of Turbulent
}

\author{
Gu Yunqing, ${ }^{1,2}$ Liu Tao, ${ }^{1}$ Mu Jiegang, ${ }^{1}$ Shi Zhengzan, ${ }^{1}$ and Zhou Peijian ${ }^{1}$ \\ ${ }^{1}$ College of Mechanical Engineering, Zhejiang University of Technology, Hangzhou 310014, China \\ ${ }^{2}$ School of Energy and Power Engineering, Jiangsu University, Jiangsu, Zhenjiang 212013, China \\ Correspondence should be addressed to Gu Yunqing; guyunqing@zjut.edu.cn
}

Received 30 April 2017; Accepted 20 August 2017; Published 18 September 2017

Academic Editor: Saurabh Das

Copyright (C) $2017 \mathrm{Gu}$ Yunqing et al. This is an open access article distributed under the Creative Commons Attribution License, which permits unrestricted use, distribution, and reproduction in any medium, provided the original work is properly cited.

\begin{abstract}
Turbulent flow is a difficult issue in fluid dynamics, the rules of which have not been totally revealed up to now. Fluid in turbulent state will result in a greater frictional force, which must consume great energy. Therefore, it is not only an important influence in saving energy and improving energy utilization rate but also an extensive application prospect in many fields, such as ship domain and aerospace. Firstly, bionic drag reduction technology is reviewed and is a hot research issue now, the drag reduction mechanism of body surface structure is analyzed, such as sharks, earthworms, and dolphins. Besides, we make a thorough study of drag reduction characteristics and mechanisms of microgrooved surface and compliant wall. Then, the relevant drag reduction technologies and mechanisms are discussed, focusing on the microbubbles, the vibrant flexible wall, the coating, the polymer drag reduction additives, superhydrophobic surface, jet surface, traveling wave surface drag reduction, and the composite drag reduction methods. Finally, applications and advancements of the drag reduction technology in turbulence are prospected.
\end{abstract}

\section{Introduction}

With the rapid increase of energy consumption, energy problem has been a global issue, which must be faced with and solved. Energy conservation and consumption reduction become an important research project at home and abroad. Drag reduction technology is urgently needed in transportation and military fields to reach the energy-saving goal. It is indicated by investigation and research that when the ship is sailing under water, the frictional force of its surface can account for $70 \% \sim 80 \%$ of total resistance. Even in the case of high-speed operation, its frictional force can also account for about $40 \%$ [1]. It can be calculated that in the case of certain power and energy, when the resistance decreases by $10 \%$, the ship's speed and distance will increase by about $3.57 \%$ [2]. When the Reynolds of fluid in a round pipe reaches $10^{5}$ and the turbulence intensity is $10 \%$, Reynolds turbulent stress will be about 100 times as much as laminar viscous stress and the greater the stress is, the greater the loss of energy is, there will be a lot of energy consuming in the flow friction [3]. In a sense, lot of energy consumption will be saved every year, even by slightly reducing the sailing resistance under water, which is of great significance for easing the energy crisis in the case of relative energy shortages nowadays.

Based on this, people always devote themselves to researching drag reduction methods. Natural creatures form a series of biological structure adapting to drag reduction after years of evolution. In recent years, scientists have found that the surface structures of many creatures are nonsmooth, the skins of sharks and dolphins have distributed many flakelike rib structures [4], which can change the skin's surface structure of turbulent layer and velocity distribution when the shark is swimming [5], while the earthworm's back can secrete some milk-white liquids, thus having wetting function and being able to reduce soil adhesion to the animal's body surface, which can research the goal of drag reduction [6]. These conditions of drag reduction on natural creatures give us great insights. Additionally, people also reach and develop many drag reduction methods, which provide many kinds of ways and means, attaining a series of achievements. 


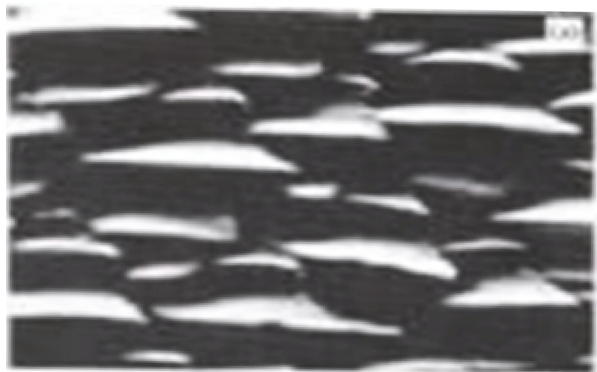

(a) Spiny dogfish skin

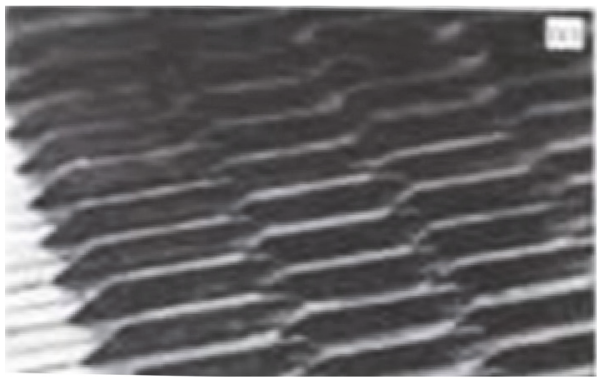

(c) Rib surface imitation of shark skin

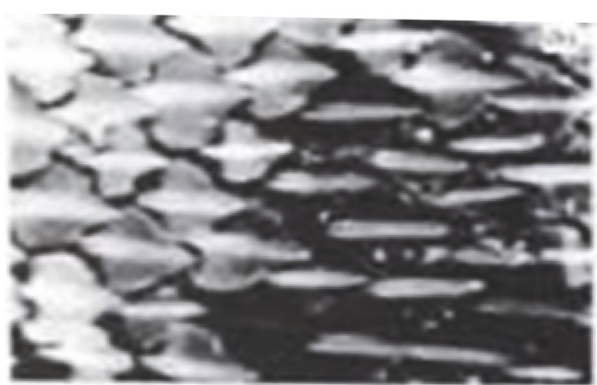

(b) Tiger shark skin

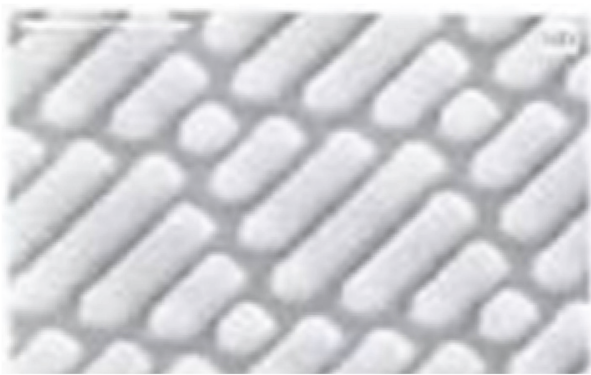

(d) Coating material imitation of shark skin

FIgURE 1: Shark skin microstructure and its imitation.

\section{Bionic Drag Reduction Technology}

2.1. Bionics. Bionics is an emerging discipline which can produce bionic productions, approximately with some characteristics of biological surfaces by imitating biological systems or using artificial technology to design and optimize productions for the biological function itself and to process for many aspects in function, shape, structure, material, and so on [7]. Natural creatures have developed into many kinds of biological bodies adapting to the environment in order to meet the need of living. These structures, shapes, and other relevant factors form the maximal ability to adapt to and coordinate the living environment by optimizing and coupling $[8,9]$. Bionics was first born in the West in 1960. At an early stage, people understood and imitated natural creatures to apply this in practical production and life. As early as 2000 years ago in China, people began to imitate natural creatures consciously, for example, the famous carpenter in ancient times, Lu ban, invented saw for lumbering by observing the jagged edges of blades grass. In today's world, bionics has undergone rapid development, some research achievements have applied in people's daily life, for example, ultrasonic testing was invented by researching the bat [10], and riblet surface drag reduction was proposed by observing the shark when swimming [11].

\subsection{Biological Surface Structures}

2.2.1. Shark Skin's Nonsmooth Surface. The shark is one of the fastest swimming animals in the ocean, whose skin surface does not adhere to any halobios, having an excellent drag reduction effect. By long-term observation for sharkskin, its surface is not smooth but is composed of many scales with grooved shape, with more spines and setae [12].
Sharkskin's microstructure and its mimic are shown in Figure 1 [13]. The scales of sharkskin are shield scales, whose configuration are compact, orderly, and jagged. The middle teeth are long, while the side teeth are short. The tooth spaces are toward the shark's tail direction and have overlapping phenomenon. Lot of researches reveal that the special structure of sharkskin mainly has two aspects of the role: on the one hand, this structure has antifouling function, decreasing adhesion on skin by other benthos; on the other hand, it is in favor of drag reduction in swimming. The cause of drag reduction is that this structure can change the intrinsic structure and the velocity distribution in turbulent boundary layer when the shark is swimming, which has a good effect on drag reduction.

2.2.2. Earthworm's Nonsmooth Skin. The earthworm is a kind of soft animal, which has the characteristic of reducing adhesion to move freely without soil adhesion in clay soil. Through the biomechanical angle of earthworm crawling, the frictional resistance between the body and the soil surface was analyzed. The characteristic of an earthworm is the result of coupling with many factors, for example, earthworm's nonsmooth skin, its soft figure, and special way of movement $[14,15]$. Earthworm's skin structure is shown in Figure 2 [6]. The back hole of the earthworm is concave pit on a nonsmooth surface structure. It can secrete some milk-white liquids and wet the surroundings, which can form a lubricated interface between soil and earthworm's body, reducing the adhesion of soil for the creature's skin.

2.2.3. Dolphin's Flexible Body Surface. The mystery of highspeed and low resistance, when the dolphin is swimming, is always one of the hot research issues for all countries' 


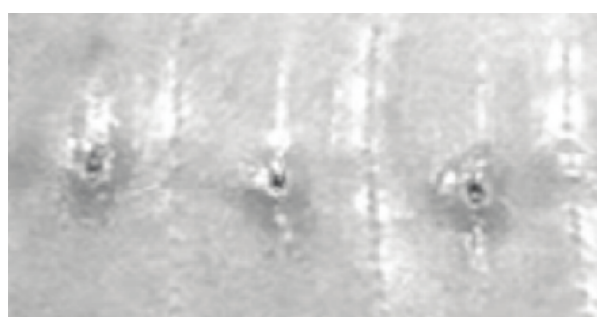

(a) Hole structure on back

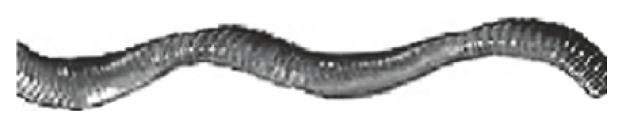

(b) Walking pattern

FIGURE 2: Earthworm's body surface.

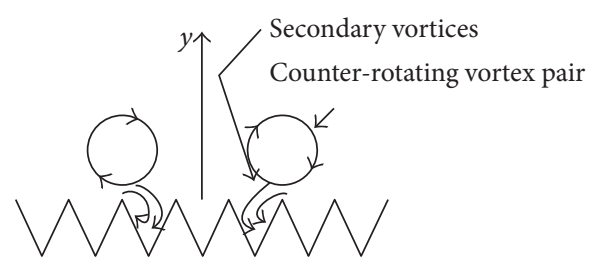

(a) Schematic diagram of vertical flow

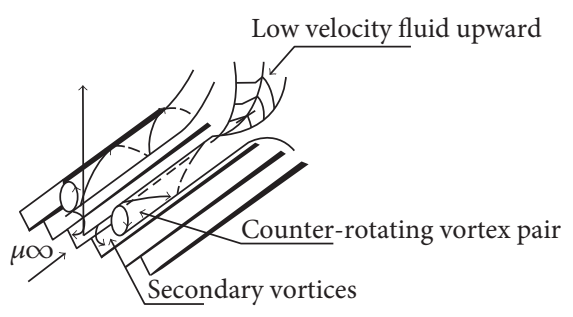

(b) Stereo diagram

FIGURE 3: Schematic diagram of the interaction between the grooved surface and streamwise vortex.

scientists. The United States, Europe, and other countries have conducted plenty of theoretical studies and experimental researches, currently attempting to find the drag reduction mechanism of the dolphin's skin, which is mainly composed of two parts by research: for one thing, in the process of dolphin's swimming, its eyes can secrete a special liquid, which covers the whole skin coordinating with the streamline body shape carve of the dolphin. The liquid can reduce the friction force when the dolphin is swimming, having a lubricating function; another most key cause is that the dolphin can produce a wave in the process of swimming. It is produced from the dolphin's head, transferring to the tail at a very high frequency and minimum amplitude, changing the dolphin's skin into a flexible skin, which is very similar with the mechanism of flexible wall drag reduction. What is more, the dolphin's skin wave can produce vortex layer when the dolphin is swimming, which has an effect on drag reduction to some extent [16].

\subsection{Bionic Drag Reduction Methods}

2.3.1. Groove Surface Drag Reduction. In the field of traditional thought, people always focus more energy on the smooth surface drag reduction. With the further research of bionics, people find that the microgrooved surface can effectively reduce the frictional force on the wall. At present, the practical applications of groove surface drag reduction mainly focus on 3 parts: aircraft, fluid drive apparatus, and pipeline fluid conveying equipment [17]. As early as the 1980s, German aircraft manufacturers began to use this technology. They found that through the technology, $8 \%$ of the fuel could save, when the plane flew the same distance. Currently, the United States, Europe, and other countries study deeply the groove surface drag reduction when the aircraft is flying and achieves great development.
By researching the groove surface drag reduction, there are mainly two aspects that influence its characteristic: its characteristic and the flow-field environment. The selffactor of groove surface includes its shape and size. The flow-field environment includes its pressure gradient, the shape of flow cross-section, and the fluid velocity. Researching a variety of geometries of groove (including $\mathrm{V}$-shape, oval, semicircular, jug, and rectangular), the drag reduction effect of the V-shape groove is the best [18]. In the study of the placement of groove surface, the surface in distribution of the flow direction can effectively control the quantity of the low strip, while the distribution of lateral direction can inhibit the length of the low strip. There is a big difference between researchers about the influence of placement mode on resistance, and the result of experiment still has a big discrepancy.

The optimal drag reduction surface is not the smooth surface as is described by classic experiments [17]. Groove surface drag reduction is related to the turbulent flowing structure. We can make a summary as follows by researching its mechanism at present; considering wall shear stress simply from fluid mechanics perspective, the groove surface drag reduction mechanism owes to the increase of the vicious sublayer thickness. The surface has an obvious delay in the transition from laminar boundary layer to turbulent boundary layer [19], which can also change the turbulent characteristics in the near wall region [20-22]. From the theory of turbulent coherent structure, its mechanism is that the streamwise vortexes associated with low-speed strips are reduced and the spanwise gather of the low-speed strips is suppressed under the interaction between streamwise vortexes and secondary eddies, as shown in Figure 3, which is produced under the groove. The strip transition on the groove surface is more flat compared with the smooth surface. Low-speed strip transitions have a good linearity, revealing that the groove restrains the lateral 
fluid flow and strengthens the stability of fluid flow. From the principle of mechanical drag reduction, a similar "air bearing theory" can be put forward.

2.3.2. Flexible Wall Drag Reduction. After a long-term study of dolphins and sharks, people find these marine organisms' skins are heavily elastic, which can reduce drug while they are swimming. According to this characteristic, scientists invent the flexible wall drag reduction method, daubing polymers on the solid wall and filling in their space with liquid to buffer external pressure with high elasticity. Both Cooper and Carpenter devote themselves to optimizing compliant coating to attain the best effect on drag reduction [22]. Russian scholars, Kulick and Semonov prove that the flexible wall has an important influence in reducing flow noise and surface frictional force by researching, the biggest drop up to $7 \%$.

The flexible wall drag reduction mechanism is mostly increasing the thickness of the wall viscous sublayer, putting off the transition from laminar boundary layer to turbulent boundary layer and reducing the velocity gradient on the boundary layer, which results in reducing the shear force on the solid wall [23]. Even though, it still has a certain limitation, which is applicable to the high-speed and turbulent state. Its witnesses are exposed in low-speed flowing. Applying the flexible wall in the low-speed flow, it can reduce its own weight and deepen the depth of ship into the water. In this condition, its effect on drag reduction is not ideal.

\section{Other Drag Reaction Methods}

3.1. Microbubble Drag Reduction. Microdouble drag reduction is one of the main approaches in achieving jet drag reduction. With the depth of microair bubble drag reduction, it mainly applies in many fields, such as ship drag reduction, ultrasonic imaging, and sewage disposal. It is worth mentioning that microbubbles have an increasing effect on ship drag reduction, which becomes one of hot research issues. For a sailing ship, drag reduction can be achieved by covering the bubble layer on the hull. Reducing the density of the fluid medium with this method can change the boundary internal structure and the flowing kinematic and dynamic characteristics of the fluid in the near wall region, as shown in Figure 4. Meanwhile, we can take advantage of the small frictional force on the bubble surface and deformable regulating flow structure to reduce drag $[24,25]$. Studies have shown that the drag reduction efficiency is up to $20 \% \sim 80 \%$. Its mechanism is very complicated and affected by many factors, such as the number of hull surface, the size of bubbles, the structure of the hull, and flow parameter on the hull surface, which can affect the drag reduction effect [24]. For the hull surface, a gas-liquid mixture can be formed by injecting air, which can change the internal structure. Because of the volatility of the bubbles, partial work, produced by applying the shear force in the fluid, translates into deformation energy, reducing the loss of energy to the drag reduction effect.

Presently, about the study of micro bubbles, the foreign scholar, Madavan et al., [26] researches the factors of influence in the microbubble drag reduction. Merkle researches the microbubble turbulent boundary layer effect of plat.

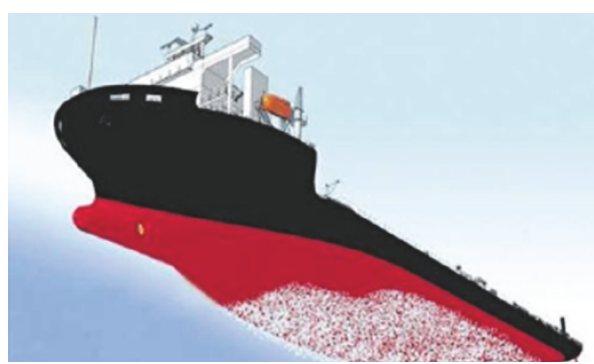

Figure 4: Bubble reduction ship.

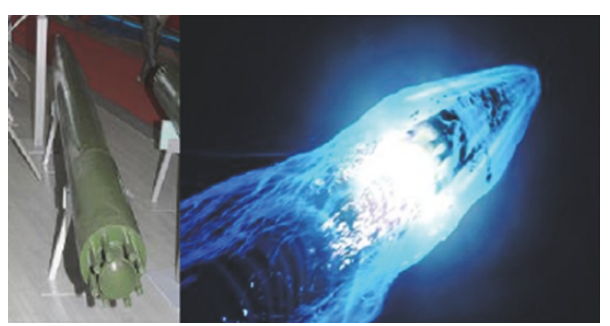

FIGURE 5: Supercavitating missile.

The study relatively lags behind and starts late in our country. Based on the achievements of his predecessors, Wang [27] researches the theory of microbubble drag reduction mechanism using the Mac method axisymmetric body in computational fluid dynamics. Now, using the technology in the field of super gun shells can reduce the resistance, thereby increasing the speed of water missile as shown in Figure 5.

Similar with flexible wall drag reduction, though microbubble drag reduction has obtained a substantial progress, it also has many weaknesses. An obvious weakness is that the bubbles covering the hull surface are not steady and vulnerable to cracks to generate enlarged drag force and noise. If the bubbles are too small, the needed drag reduction cannot be achieved [24]. Besides, its another weakness is that when the ship is sailing, the microbubbles covering on its surface are mainly hydrogen and oxygen bubbles generated by electrolyzing water. Though the source of reactant is abundant and its resultants are not pollutional to the environment, the degree of demand for and dependence on electric energy is very high.

3.2. Vibrant Wall Drag Reduction. Relative to the previous several drag reduction method, wall vibration reduction is a relatively new drag reduction technology, which refers to the exhibition along the flow direction of the fluid flow through the smooth plate and vibration, which leads to a method of flat surface resistance decreased, as shown in Figure 6. Jung et al. verified the effectiveness of drag reduction about periodic spanwise vibration of smooth flat plate in 1992, using DNS means for the first time [28]. In recent years, foreign scholars have revealed the vibrant wall drag reduction mechanism by adopting DNS computational method and analyzing DNS data. There are two drag reduction mechanisms about the vibrant wall at present: first, the 


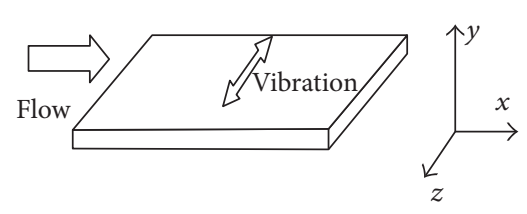

Figure 6: Schematic diagram of spanwise wall oscillation.

strips and vortexes generate an obvious incline, which can produce negative spanwise vortexes decreasing the mean velocity gradient in viscous sublayer and influencing the structure of the turbulent boundary layer. The streamwise vortexes rearrange along the spanwise, weakening the wave intensity of the streamwise vortexes in the lateral boundary layer, which is in favor of drag reduction. Another theory is that wall vibration interferes with the regenerative cycle of the quasi-streamwise vortexes, not sustaining wall turbulence, which can achieve the effect of drag reduction [22]. Because of the complexity of turbulence, there is no accurate expression about wall drag reduction at present and relevant researches need to be done in the future.

3.3. Coating Drag Reduction. With the rapid development of the economy, the demand for crude oil is increasing. Pipeline construction is imminent. In the long distance transportation of oil process, the friction drag between the oil and pipeline accounts for $98 \%$ of the total drag, which makes the power of the pump station almost entirely overcomes friction [29]. If the transportation drag of oil can be reduced, it can bring huge economic benefits. Based on this, the drag reduction problem in the pipeline has always puzzled researchers. With the development of coatings technology, coating in the pipeline as a mature technology has been applied in the long distance transportation of oil. The application of the coating in the pipeline can not only reduce the resistance, but also reduce the corrosion of the oil pipeline. As early as 1953, this method was applied in gas transportation in trunk pipeline of oil, which can increase the gas transportation capacity by $5 \% \sim 20 \%$ [30]. Wei and Ni discover the drag reduction effect of silicone oil is the best. The reason is the hydrophobicity of silicone oil can make the wall more smooth [29]. The hydrophobicity of coating and control for wall roughness is the essential reason of coating drag reduction.

The coating drag reduction mechanism mainly focuses on two aspects: smooth surface drag reduction and low surface energy drag reduction. In the pipeline process, because of the limitation of working accuracy, the pipeline always presents a certain degree of roughness and its surface is rugged. The flowing of oil in the pipeline is in turbulent state, which can form vortex area on the surfaces of concaveconvex objects which can generate a certain pressure, bringing loss of energy which is related to the mean height of concave-convex objects. The higher the mean height is, the greater the loss of energy is. The coating on the tube wall can effectively smooth the wall to reduce the concaveconvex height, which can reduce the mechanical drag produced by wall roughness. It is discovered by foreign relevant researches that the transition from laminar boundary layer to turbulent boundary layer can be put off by spraying compliant coating. For the low surface energy drag reduction, spraying compliant coating changes the wetting degree of the fluid on the tube wall because of the hydrophobicity of coating, reduces the velocity gradient of the fluid on the tube wall and, decreases the shear force on the wall, which can reach the goal of reducing the transportation drag. What is more, the difficulty that coating can be wetted is related to its drag reduction performance. The more difficult the coating can be wetted, the worse its drag reduction effect is.

3.4. Polymer Additive Drag Reduction. Besides the coating drag reduction, the drag reduction purpose also can be achieved by adding the polymer in the fluid. In the late 19th century, people found that muddy water can flow faster than clean water in some river sections. Later, they found that the frictional drag on the ship's surface is smaller when its sails in the water with water plants than without water plants [31]. In the late 1940s, Toms found the earliest drag reduction agent (DRA), polymethyl methacrylate (PMMA). Afterwards, researchers found other additives one after another. The DRA can be broadly divided into two categories: water-soluble and oil-soluble. Water-soluble DRA includes sesbania power, PAM (polyacrylamide), and synthetic PEO (Polyoxyethylene). Oil-soluble DRA includes olefin copolymer and polyisobutene [32].

In our country, though the study of DRA starts relatively late, great progress has still been made. In the 1980s and early 90s, Chengdu University of Science and Technology and Zhejiang University made a remarkable advance for the synthesis of indoor DRAs. The China Petroleum Pipeline Company made a breakthrough progress for the development of EP series oils, DRA, forming the independent intellectual property rights and starting mass production.

The polymer additives drag reduction is not still mature, but certain research results have been made. In 1967, Virk et al. discovered that there exists an elastic buffer area between laminar flow and turbulent flow in the near wall area, by measuring the fluid velocity before and after adding DRA, according to which, asymptote was being obtained. Early researchers consider that the existence of the elastic buffer area results in the enlargement of velocity and increase of flow in the pipe, attaining the drag reduction effect. Besides, Berman had a new discovery that the effect is better by injecting DRA than DRA is dissolved in liquid beforehand in 1986. Focusing on the influence of fluid kinetic characteristic, the polymer can change the turbulent structure on the wall. Abernathy drew two conclusions one is that polymer can impede the generation of eddies and reduce the frequency of vortex; the other is that polymer can reduce the rotation rate of the vortex. Both of them together can result in the drag reduction effect. Some hypotheses and analyses can be proposed combining with the experimental results for the polymer drag reduction mechanism, but there is still no unified theory which can explain all experimental phenomena $[33,34]$.

At the same time, artificial polymer can cause biological damage to the ship's hull, and some organic DRA can pollute the environment. So, the polymer drag reduction agent is commonly used for oil pipeline transportation, but it will add additional funding. 


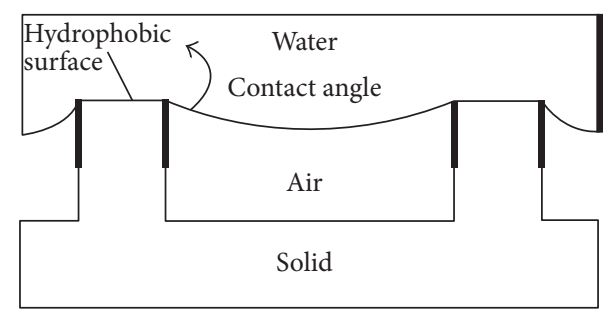

FIGURE 7: Schematic diagram of drag reduction mechanism of the superhydrophobic surface.

3.5. Superhydrophobic Surface Drag Reduction. According to the phenomenon, water rolls easily without wetting on the lotus leaf because of the microscale structure of the emulsion on the lotus leaf rejecting the water, it invented the method of the superhydrophobic surface drag reduction [35]. So far, the mechanism accepted of the superhydrophobic surface drag reduction is the theory of slip length considering that because water produces the wall slip through hydrophobic surface decreasing velocity gradient of the boundary layer, decreasing the shear stress, and delaying the change of laminar attachment surface, the laminar flow regime tends to be more stable [36]. The mechanism of the superhydrophobic surface drag reduction is shown in Figure 7.

Voronov et al. [37] found that by numerical simulation, while the slip length and contact angle are usually regarded as hydrophobic performance standards, they do not always follow the same rules, low $\sigma_{\mathrm{r}}$ (relative atomic size) tends to make the contact while the angle increases but will reduce the slip length. Bixler and Bhushan [38] obtained a maximum drag reduction rate of $26 \%$ by simulating shark skin resistance reduction experiments. Lee and Kim [39] had studied the applicable range of superhydrophobic drag reduction on hydraulic pressure. The present study found that the texture of the superhydrophobic surface captures the air to form the air cushion and reduces the frictional resistance between the fluid and the coating surface. Using the drag reduction characteristics of the superhydrophobic surface, the technique is applied to the surface of the swimming suit. As shown in Figure 8, this swimsuit is more conducive to increasing the speed of far mobilization compared to the imitation shark skin swimsuit.

3.6. Jet Surface Drag Reduction. The aim of jet technology is to control and provide power for itself through the interaction force of jet reaction and the interaction between jet and flow. It has short response time and good low voltage control characteristics [40].

Sharks living in the sea are aquatic animals having rapid movement. The burst start speed of the deep-sea sharks is amazing, reaching up to $10 \sim 20 \mathrm{~m} / \mathrm{s}$; while pursuing their prey, they have very fast speed [41]. There are large gill plates lined in front the body side of sharks, as shown in Figure 9, and each lamella has about $5 \sim 7$ branchial cleft. As they swim, the water is sucked through the half opening mouth, and gas is exchanged from the gill slits. It is closely related with breathing and self-motion resistance reduction.
The researchers found the drag reduction effect on the surface of the jet according to the jet motion of the shark's gills. The present study generally believes that if there is jet on the motion face, jet fluid will block the mainstream field, forming the countercurrent area at the back of the jet hole and the direction of speed near the wall is contrary to flow velocity in the countercurrent area having the obvious drag reduction effect. Furthermore, there is the counter-rotating vortex extending downstream produced in the downstream of the jet hole and it would induce two vortex on the wall increasing the thickness of the boundary layer and decreasing the velocity gradient both having the effect of drag reduction [42].

To sum up, with the rapid development of science and technology, jet drag reduction technology has reached rapid development and fruitful achievements, many research results have been applied in the engineering practice, but there are still many problems that still need further research.

3.7. Traveling Wave Surface Drag Reduction. Traveling wave surface drag reduction technology is considered a very promising drag reduction technology and is made of corrugated shape, which was inspired from the undulating dunes structure of desert, as shown in Figure 10. At present, the mechanism of drag reduction for traveling wave surface is still controversial. A more persuasive argument is that the ripples on the surface of the traveling wave shape can produce secondary flow, namely the origin parallel artificial vortex flow sparked a row, so that it is free to flow in the parallel artificial vortex flow, achieving the goal of drag and noise reduction. Its principle is shown in Figure 11.

3.8. Combined Drag Reduction. In practical engineering application, multiple drag reduction technologies would be used together achieving the best drag reduction effect. The effect of drag reduction using multiple drag reduction technologies is generally better than that using single drag reduction technique. It is the most universal using trench surface drag reduction and other drag reduction methods together in a variety of combinations. A series of turbulent drag reduction tests on coupling of trench surface and polymer coating technology have been carried out turning out that the effect of coupled drag reduction is excellent for the trench surface drag reduction and polymer coating drag reduction. Sun and found that this combination increases the stability of the wall air layer can make bubble not easy to break reducing the resistance and noise, so that the effect of drag reduction is optimized [11].

\section{Summary and Outlook}

The turbulent drag reduction technique a case turbulence theory applied to engineering has good development prospects. Due to the need of practical engineering, Chinese and foreigners have carried out a large number of experimental studies and gotten many achievements as references for practical application. However, most studies still stay in the simulation and test phase having not forming the mature systemic theory. In future research, the exploration of drag 

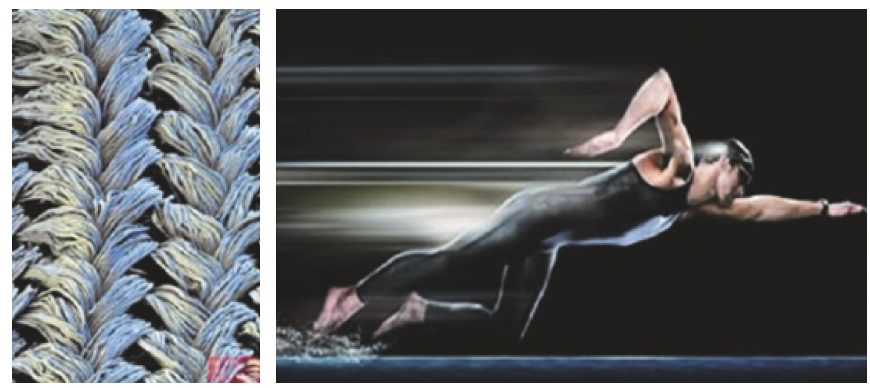

FIGURE 8: Bionic swimsuit.

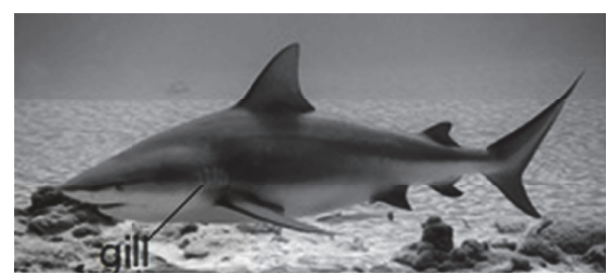

Figure 9: Feature of shark gills.

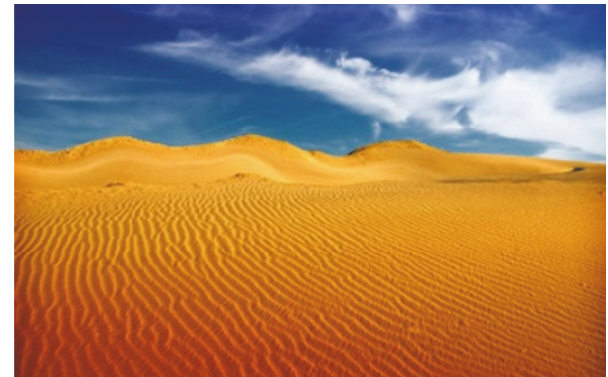

Figure 10: Wavy structures in the desert.

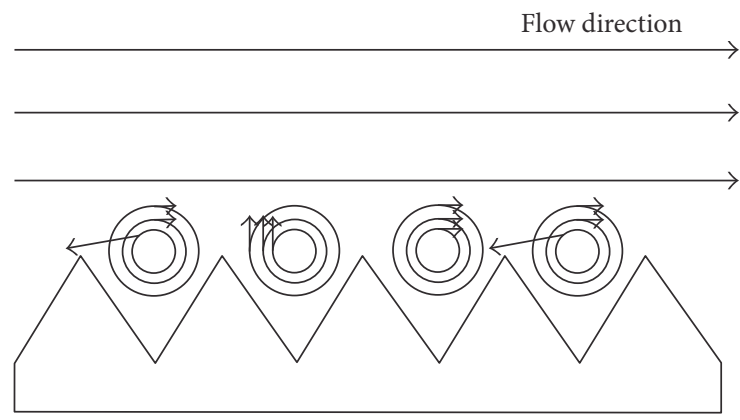

FIgURE 11: Schematic diagram of traveling wave.

reduction mechanism should be put into more important place, especially by combining the numerical simulation and experimental study and focus on theoretical research guiding practice with theory. The drag reduction effect is greatly restricted, due to that, the research work of the current technology of drag reduction is mainly focused on single drag reduction technology. Therefore, to use multiple drag reduction technologies together will be an important direction for the future research. The research of technology is service for practice, so we should speed up the progress of promoting laboratory research results to the engineering application. And how to put the relatively mature drag reduction technology into actual projects and to promote it is also an important direction in the future research.

\section{Conflicts of Interest}

The authors declare that they have no conflicts of interest.

\section{Acknowledgments}

This project is supported by the National Natural Science Foundation of China (51779226, 51476144), the Zhejiang Provincial Natural Science Foundation of China (LQ15E050005), the Public Welfare Technology Application Projects of Zhejiang Province (2017C31025), and the China Postdoctoral Science Foundation (2016M601736).

\section{References}

[1] Y. B. Li, Y. Q. Gu, J. G. Mou, S. H. Zheng, and L. F. Jiang, "Current research status of biomimetic drag-reducing antifouling paints with low surface energy," Materials Protection, vol. 47, no. 6 , pp. $48-51,2014$.

[2] J. L. Xu, X. R. Lai, B. Wang, L. Xu, and G. H. Liu, "Flow patterns and parameters of micro bubble generation in a flow focusing device," Nanotechnology and Precision Engineering, vol. 10, no. 1, pp. 59-63, 2012.

[3] J. F. Sun, Y. Zhou, J. Li, X. S. Liu, and J. Y. Lan, "The design of testing equipment for drag resistance effect based on differential principle and the study of drag reduction characteristic of coatings," Lubrication Engineering, vol. 7, pp. 120-122, 2006.

[4] Y. R. Sun and Z. D. Dai, "Bionics today and tomorrow," Acta Biophysica Sinica, vol. 23, no. 2, pp. 109-114, 2007.

[5] Z. Y. Zhao and S. P. Dong, "Application of micro-grooved surface for turbulent drag reduction," Journal of Petrochemical Universities, vol. 17, no. 3, pp. 76-79, 2004.

[6] Y. Q. Gu, J. G. Mou, D. S. Dai et al., "Characteristics on drag reduction of bionic jet surface based on earthworm's back orifice jet," Acta Physica Sinica, vol. 64, no. 2, article 024701, 2015.

[7] Y. X. Lu, "Significance and progress of bionics," Journal of Bionic Engineering, vol. 1, no. 1, pp. 1-3, 2004. 
[8] H. Y. Wang, G. W. He, M. F. Xia, F. J. Ke, and Y. L. Bai, "Multiscale coupling in complex mechanical systems," Chemical Engineering Science, vol. 59, no. 8-9, pp. 1677-1686, 2004.

[9] E. T. Liu, "Systems biology, integrative biology, predictive biology," Cell, vol. 121, no. 4, pp. 505-506, 2005.

[10] G. Neuweiler and E. Covey, The Biology of Bats, Oxford University Press, Oxford, 2000.

[11] J. R. Sun and Z. D. Dai, "Bionics of non-smooth surface I," Progress in Natural Science, vol. 18, no. 3, pp. 241-246, 2008.

[12] P. Ball, "Engineering shark skin and other solutions," Nature, vol. 400, no. 6744, pp. 507-509, 1999.

[13] J. R. Sun and Z. D. Dai, "Bionics of non-smooth surface II," Progress in Natural Science, vol. 18, no. 7, pp. 727-733, 2008.

[14] T. Shelley, "Worms show way to efficiently move," Eureka, vol. 24, no. 1, pp. 28-29, 2004.

[15] L. Q. Ren, J. Tong, J. Q. Li, and B. C. Chen, "Soil adhesion and biomimetics of soil-engaging components: a review," Journal of Agricultural Engineering Research, vol. 79, no. 3, pp. 239263, 2001.

[16] F. E. Fish, "The myth and reality of Gray's paradox: implication of dolphin drag reduction for technology," Bioinspiration \& Biomimetics, vol. 1, no. 2, pp. R17-R25, 2006.

[17] D. B. Huang, X. H. Deng, and Y. J. Wang, "Numerical simulation study of turbulent drag reduction over ribelt surfaces of tubes," Journal of Hydrodynamics, Series A, vol. 20, no. 1, pp. 101-105, 2005.

[18] K. S. Chio, "European drag-reduction research-recent developments and current status," Fluid Dynamics Research, vol. 26, no. 5, pp. 325-330, 2000.

[19] J. J. Wang, S. L. Lan, and F. Y. Miao, "Drag-reduction characteristics of turbulent boundary layer flow over riblets surfaces," Shipbuilding of China, vol. 42, no. 4, pp. 1-5, 2001.

[20] H. W. Yang and G. Gao, "Experimental study for turbulent drag reduction using a novel boundary control technique," Acta Aeronautica Et Astronautica Sinica, vol. 18, no. 4, pp. 455-457, 1997.

[21] Y. B. Li, Z. D. Qiao, and Z. Q. Wang, "An experimental research of drag reduction using riblets for the Y-7 airplane," Aerodynamic Experiment and Measurement \& Control, vol. 9, no. 3, pp. 21-26, 1995.

[22] A. J. Cooper and P. W. Carpenter, "The stability of rotatingdisc boundary-layer flow over a compliant wall. Part 2. Absolute instability," Journal of Fluid Mechanics, vol. 350, pp. 261-270, 1997.

[23] Y. Yao, C. J. Lu, T. Si, and K. Zhu, "Water tunnel experimental investigation on the drag reduction characteristics of the traveling wavy wall," Journal of Hydrodynamics, Series B, vol. 23, no. 1, pp. 65-70, 2011.

[24] F. Guo, W. C. Dong, and Y. Bi, "The theoretical study on influencing factors to resistance reduction of a gyroidal object by microbubbles," Journal of Harbin Engineering University, vol. 31, no. 11, pp. 1443-1449, 2010.

[25] K. Mohanarangam, S. C. P. Cheung, J. Y. Tu, and L. Chen, "Numerical simulation of micro-bubble drag reduction using population balance model," Ocean Engineering, vol. 36, no. 11, pp. 863-872, 2009.

[26] N. K. Madavan, S. Deutsch, and C. L. Merkle, "Reduction of turbulent skin friction by microbubbles," Physics of Fluids, vol. 27, no. 2, pp. 356-363, 1984.
[27] J. Q. Wang, Elementary Study on Wall Shin Friction Reduction of Symmetrical Body by Microbubbles in Boundary Layer of Fluid, Tianjin University, Tianjin, 2004.

[28] A. Baron and M. Quadrio, "Turbulent drag reduction by spanwise wall oscillations," Applied Scientific Research, vol. 55, pp. 311-326, 1996.

[29] J. T. Wei and B. Ni, "The drag reduction research of the additives in the heating network," Journal of Changchun Institute of Technology, vol. 12, no. 3, pp. 48-51, 2011.

[30] D. W. Bechert, M. Bruse, W. V. Hage, J. T. Van der Hoeven, and G. Hoppe, "Experiments on drag-reducing surfaces and their optimization with an adjustable geometry," Journal of Fluid Mechanics, vol. 338, no. 5, pp. 59-87, 1997.

[31] Y. R. Qun and Q. Si, "Study on heat transfer and drag reduction in flow boiling of polymer additives," Chemical Engineering, vol. 29, no. 5, pp. 22-25, 2001.

[32] H. L. Petrie, S. Deutsch, T. A. Brungart, and A. A. Fontain, "Polymer drag reduction with surface roughness in flat-plate turbulent boundary layer flow," Experiments in Fluids, vol. 35, no. 1, pp. 8-23, 2003.

[33] K. S. Choi, "Near-wall structure of a turbulent boundary layer with riblets," Journal of Fluid Mechanics, vol. 208, no. 6, pp. 417-458, 1989.

[34] K. W. Li and Q. X. Lian, "A review on drag reduction of boundary layer," Journal of Beijing Uinversity of Aeronautics and Astronautics, vol. 4, pp. 68-76, 1991.

[35] X. Q. Hao, L. Wang, Y. C. Ding, G. H. Ye, Z. Y. He, and B. H. $\mathrm{Lu}$, "Study on the dray reduction of the superhydrophobic surface," Lubrication Engineering, vol. 34, no. 9, pp. 2528, 2009.

[36] X. L. Wang, Q. F. Di, R. L. Zhang, and C. Y. Gu, "Surface slip effect and its application to drag reduction technology," Advances in Mechanics, vol. 40, no. 3, pp. 241-249, 2010.

[37] R. S. Voronov, D. V. Papavassiliou, and L. L. Lee, "Slip length and contact angle over hydrophobic surfaces," Chemical Physics Letters, vol. 441, no. 4-6, pp. 273-276, 2007.

[38] G. D. Bixler and B. Bhushan, "Shark skin inspired low-drag microstructured surfaces in closed channel flow," Journal of Colloid \& Interface Science, vol. 393, no. 1, p. 384, 2013.

[39] C. Lee and C. J. Kim, "Underwater restoration and retention of gases on superhydrophobic surfaces for drag reduction," Physical Review Letters, vol. 106, no. 1, article 014502, 2011.

[40] B. Srivastava, "Lateral jet control of a supersonic missile: computational and experimental comparisons," Journal of Spacecraft \& Rockets, vol. 35, no. 2, pp. 140-146, 1998.

[41] W. F. Reif, "Morphogenesis and function of the squamation in sharks," Neues Jahrbuch für Geologie und Paläontologie, Abhandlungen, vol. 164, pp. 172-183, 1982.

[42] F. Li, G. Zhao, W. X. Liu, and Z. Z. Sun, "Simulation on flow control and drag reduction with bionic jet surface," Journal of Basic Science and Engineering, vol. 22, no. 3, pp. 574-583, 2014. 


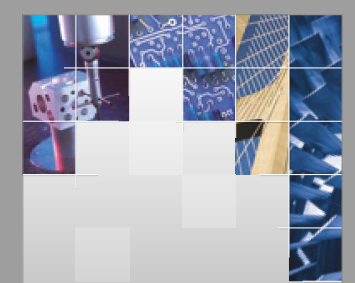

\section{Enfincering}
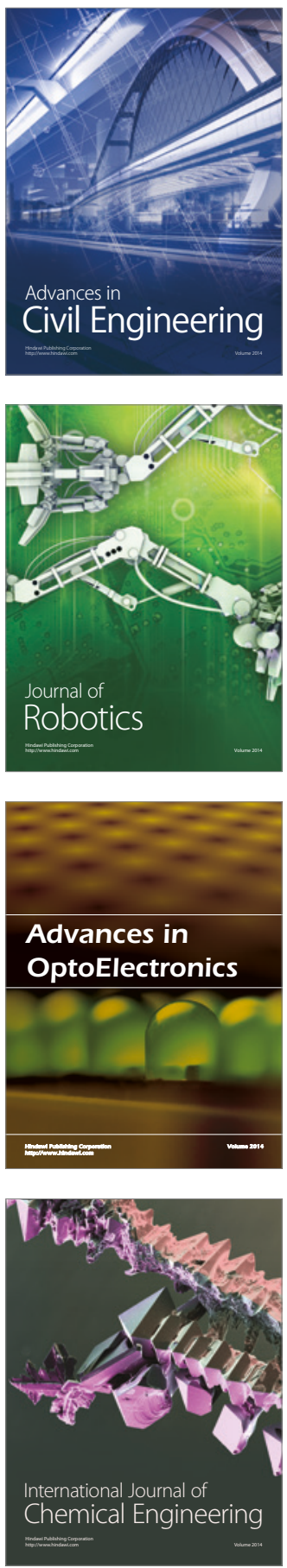

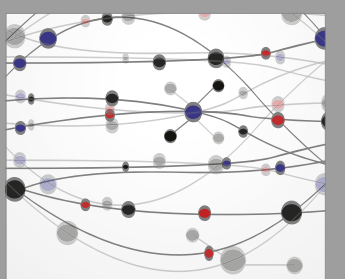

The Scientific World Journal

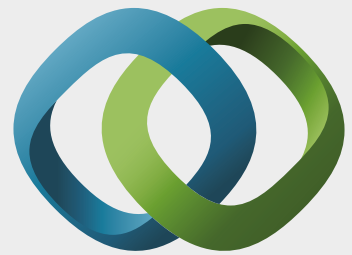

\section{Hindawi}

Submit your manuscripts at

https://www.hindawi.com
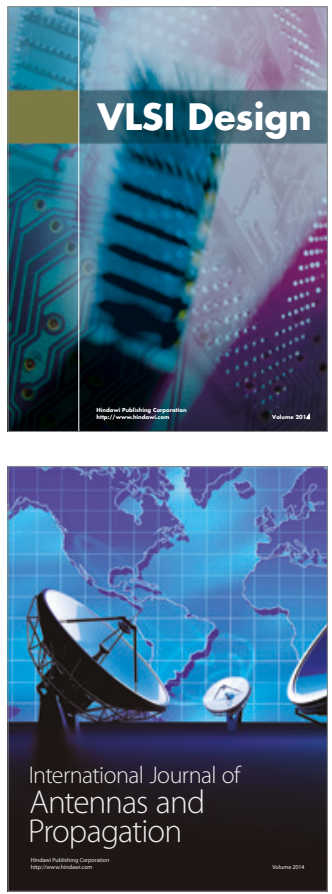

\section{Rotating}

Machinery
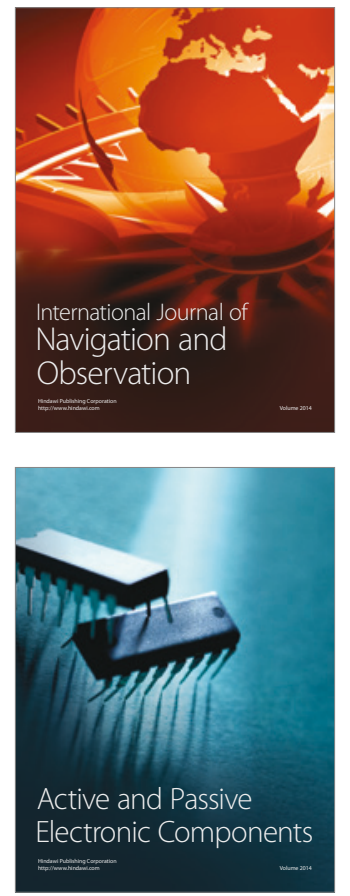
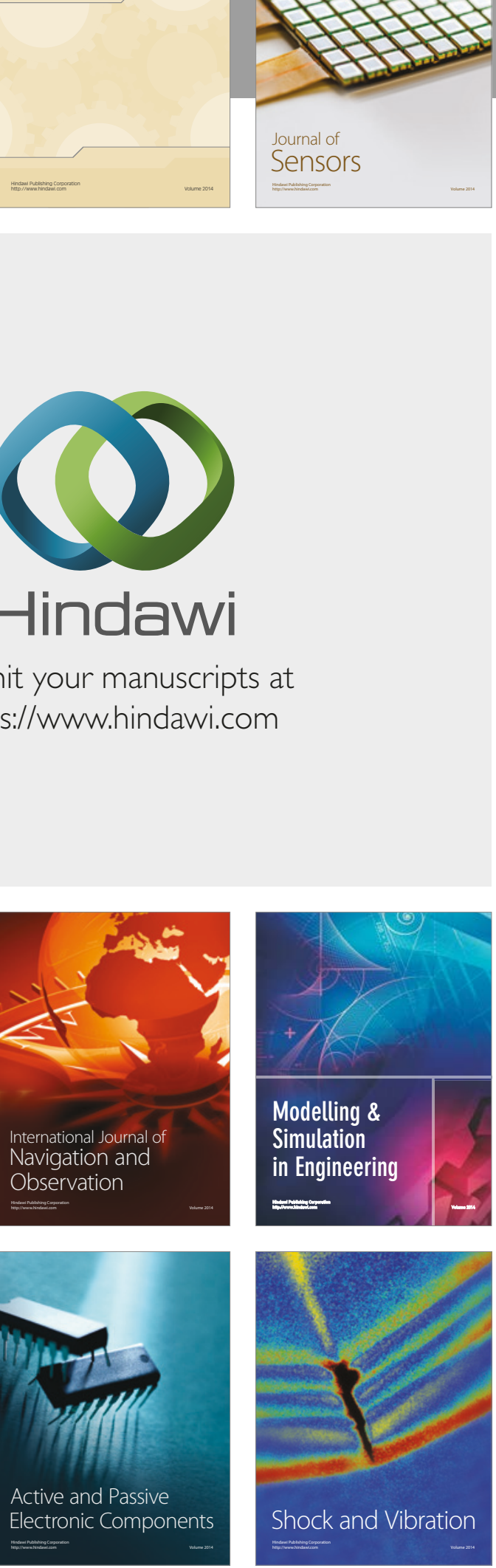
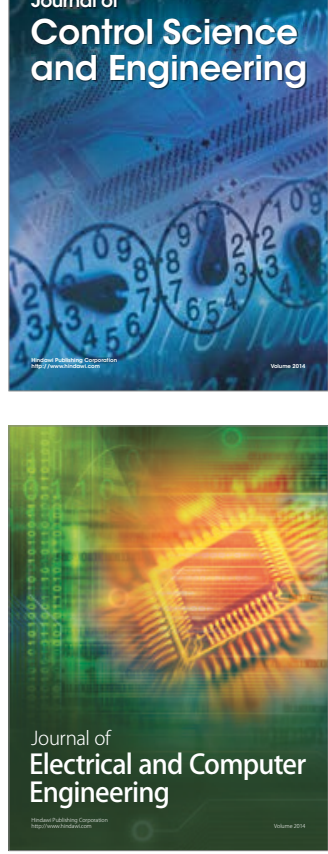

Distributed

Journal of

Control Science

and Engineering
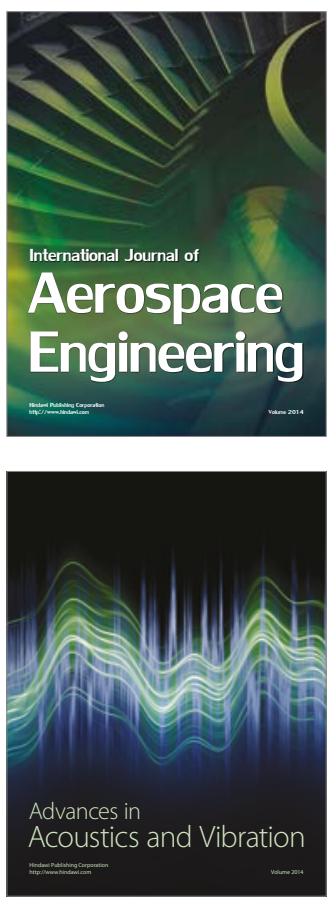

Sensor Networks 